in-hospital mortality and ischemic stroke or death (adjusted odds ratios 0.25 [95\% $\mathrm{Cl} 0.07-0.90$ ] and 0.55 [95\% Cl 0.32-0.95], respectively), but was not associated with the frequency of in-hospital cardiac outcomes. In asymptomatic patients, statin use did not affect occurrence of ischemic stroke or death or in-hospital mortality, which could reflect the low frequency of outcomes observed in the asymptomatic patient group; a protective effect might have been observed with a larger sample size.

The mechanisms of statin action are yet to be determined. Randomized controlled trials are required to confirm the suggested protective effect of statin therapy in symptomatic patients undergoing carotid endarterectomy.

Kate Matthews

Original article Kennedy J et al. (2005) Statins are associated with better outcomes after carotid endarterectomy in symptomatic patients. Stroke 36: 2072-2076

\section{Antipsychotics upregulate lipid biosynthetic genes}

People with schizophrenia are reported to show oligodendrocyte dysfunction and downregulation of genes involved in brain myelination. Glialcell-derived cholesterol is known to be essential for CNS myelination and synaptogenesis, and a recent study by Ferno and colleagues in Norway examined whether the effectiveness of the antipsychotic drugs clozapine and haloperidol might be related to their ability to combat the decreased myelination and lipogenesis in schizophrenic patients.

The researchers used microarray analysis to identify changes in the global gene expression profile of cultured human glioma cells exposed for 24 hours to haloperidol, clozapine or vehicle. The drugs were found to upregulate a cluster of genes encoding cholesterol and fatty acid biosynthetic enzymes, the transcription of which is controlled by the sterol regulatory element-binding proteins SREBP-1 and SREBP-2. Both drugs were also found to activate the SREBP system. Clozapine was considerably more potent at activating the SREBP proteins than haloperidol in the context of therapeutically relevant concentrations, and the former also showed greater therapeutic efficacy, but it is not yet clear whether these two observations are linked.
The authors conclude that stimulation of cholesterol and fatty acid biosynthesis in glial cells might represent a novel mechanism of action for clozapine and haloperidol. While acknowledging that their study was conducted using cell culture only, they suggest that clozapine might be potent enough to produce these effects in vivo, which could have relevance for the mechanisms of antipsychoticassociated weight gain, dyslipidemia and type Il diabetes in schizophrenic patients.

Christine Kyme

Original article Ferno J et al. (2005) Antipsychotic drugs activate SREBP-regulated expression of lipid biosynthetic genes in cultured human glioma cells: a novel mechanism of action? Pharmacogenomics J 5: 298-304

\section{Prevention of cluster headache: a new study}

To avoid the severe pain associated with cluster headache, sufferers often seek preventive therapies such as oral steroids; however, these treatments can lead to steroid dependency and side effects. There is some evidence that a 'single shot' approach, based on suboccipital steroid injection, can provide long-term relief in these patients. Until now, however, no robust data have been available to support these observations. Ambrosini and colleagues have sought to address this issue in their recent double-blind, placebo-controlled trial, the results of which have been published in the journal Pain.

The team randomized 23 patients with episodic or chronic cluster headache to receive an injection of either a betamethasone preparation or physiological saline. In each case the injection was given in the region of the greater occipital nerve, on the same side as the pain. One week after the injection, 11 of the 13 patients who received active treatment $(85 \%)$ were free of symptoms, whereas all of those in the placebo group continued to suffer attacks $(P=0.0001)$. The response to treatment was maintained throughout the 4-week study period in eight $(61 \%)$ of the steroid-treated patients. No adverse events were reported, other than transient pain at the injection site, which was reported in two patients who received active treatment.

The researchers now plan to compare suboccipital and intramuscular injections of the betamethasone preparation, which includes long-acting and rapid-acting formulations, to 